\title{
Artificial Intelligence: An Advancing Front of Dentistry
}

\author{
Amrita Pandita Bhatia ${ }^{1}$ and Shilpi Tiwari ${ }^{2 *}$ \\ ${ }^{1}$ Reader, Department of Prosthodontics, NIMS Dental College, Jaipur, India \\ ${ }^{2}$ Reader, Department of Pedodontics and Preventive Dentistry, People's College of Dental Sciences and Research Centre, Bhopal, India \\ *Corresponding Author: Shilpi Tiwari, Reader, Department of Pedodontics and Preventive Dentistry, People's College of Dental \\ Sciences and Research Centre, Bhopal, India.
}

Received: November 06, 2019; Published: November 27, 2019

DOI: $10.31080 /$ ASDS.2019.03.0714

\begin{abstract}
Advances in digital technology have greatly transformed our everyday lives bringing everything within the reach of our fingertips. Over the years newer technologies have developed based on principles that try to simulate the functioning of human brain. The vast search in this field has given rise to what is known as artificial intelligence (AI). AI based diagnosis is gaining importance due to its ability to detect and diagnose lesions which may go unnoticed to the human eye, thereby paving way for a holistic practice. Artificial intelligence can be a useful modality in diagnosis and treatment of lesions of oral cavity and can be employed in screening and classifying suspicious altered mucosa undergoing premalignant and malignant changes. Its various applications in different areas of dentistry has been explained thoroughly in this paper.
\end{abstract}

Keywords: Artificial Intelligence; Diagnosis; Tumors; Applications

\section{Introduction}

As we know, the world is rapidly progressing towards digitalisation in every sphere of life. This digital technology has further simplified life. This digital revolution has revolutionised dentistry in every aspect and improved the quality of dental care. The human brain is incomparable structure which is composed of networks of interlinked neurons which transmit signals throughout the body [1]. Human brain has a complex neural structure. Over the years man has tried to build technologies which can simulate functioning of human brain accurately which has led to development of technology what is known as artificial intelligence (AI). AI is defined as a field of science and engineering concerned with the computational understanding of what is commonly called intelligent behaviour and with the creation of artefacts that exhibit such behaviour [2]. The applications of AI in the field of telecommunication and aerospace like expert systems, game playing, theorem-proving, natural language processing, image recognition and robotics have grown manifold. Technology has also revolutionised in the field of Medicine and Dentistry [3]. The latest advancement in artificial neural networks can detect and diagnose lesions which can go undetected to human eye. These systems help connect dental health care professionals all over the world. With their personal smart devices, the patients can enter the symptoms that they are experiencing and can be made conscious of the most probable diagnosis of the illness. In 2009, Kim., et al.in a study explored the relationship between dental pain and daily tooth brushing frequency, tooth brushing time, experience of tooth brushing instructions, use of dental floss, tooth brush replacement cycle, receiving scaling or not and other factors including nutrition and exercise. It was concluded in the study oral hygiene was the most important factors in preventing toothaches and predictive model of toothache was developed $[4,5]$.

Role of artificial intelligence systems in dental education

AI has revolutionised the field of dental education by creating high quality training environments. Students can do their preclinical work on virtual patients instead of phantom heads or 
simulators [6]. These virtual patients are interactive and increases the efficiency of preclinical work and eliminates risk associated with working on a live patient [7].

Role of artificial intelligence in diagnosis and treatment

AI has revolutionised field of diagnosis. With simple mobile applications, pictures of lesion can be uploaded in the application and diagnosis can be made by comparing the pictures from the vast data available around the world. Thus, doctors can prioritize the appointment when necessary and patients have easy access to expert opinion.

Artificial intelligence can be useful in screening and diagnosing suspicious oral lesions into premalignant and malignant lesions.AI provides both qualitative and quantitative data based on data input to the practitioner and thus improving accuracy in early detection, diagnosis, treatment planning [7].

Artificial intelligence in detecting dental caries

AI assists in detecting dental caries on radiographs. A clinician can overlook carious lesion due to fatigue. There are many factors that can hamper diagnosis and treating caries like variations in clinical training, errors in perception, and cognitive errors of classifying health and disease. These errors can be easily overcome by utilizing AI. It can detect and highlight caries lesion on radiograph. AI-based software developed by Dentistry. AI highlights potential carious lesions on bitewing radiographs thereby helping dentists in caries diagnosis. These software's can also be developed as patient awareness tool and thereby increasing treatment acceptance [8].

\section{Role of Artificial Intelligence in Orthodontics:}

The most talked about recent innovation is in AI driven customized orthodontic treatment. AI is now available for orthodontic diagnosis, treatment planning, and treatment monitoring. With precise 3D scans and virtual models, it is easy to $3 \mathrm{D}$ print the aligners with customized treatment plan. As the vast data get computed, it creates an algorithm which in terms intelligently decides how a patient's tooth or teeth should be moved, with how much pressure, even identifying pressure points for that particular tooth or teeth. The AI aided aligners not only deliver precise treatment execution but also helps in monitoring the progress as well and claim to reduce treatment time as well as appointment schedules. The most talked about recent innovation is in AI driven customized orthodontic treatment. AI has also important role in orthodontic diagnosis, treatment planning, and treatment monitoring. There is vast advancement in $3 \mathrm{D}$ technology, teeth can be scanned and with the help of 3D printers, models can be printed. On these 3D printed models aligners are made [9]. In the software, vast data get uploaded. this data is analysed by AI and then it intelligently decides how a patient's tooth or teeth should be moved, with how much pressure, even identifying pressure points for that particular tooth or teeth. For the application of pressure resin tags are made on tooth surface. Digital impressions has ruled out use of conventional impressions and associated laboratory steps. The treatment outcome can be predicted with the analysis of software [10].

\section{Role of artificial intelligence in prosthodontics}

The significant advantage of $\mathrm{AI}$ is its ability to analyse and learn from the millions of doctor-approved crowns in the database as cases are being continuously added to the cloud every day. To learn from the successful crown designs, the computer assesses how each high-performance restoration is made to achieve optimal function, based on the ideal occlusion, contacts and margins suited for each case [11]. CAD/CAM (Computer aided design/Computer aided manufacturing) application in dentistry is the process by which finished dental restoration are obtained through fine milling process of ready ceramic blocks. It is used in manufacturing of inlays, on lays, crowns as well as crowns and bridges. CAD/CAM technique helps in scanning prepared tooth and designing of restoration with milling. Thus, fabrication of prosthetic restoration has become easy as there are no casting steps involved [12].

\section{Role of artificial intelligence in oral surgery}

The greatest application of artificial intelligence in oral surgery is the development of robotic surgery where human body motion and human intelligence is simulated [13]. AI has also played a important role in oral implant surgery, removal of tumour and foreign bodies, biopsy and TMJ surgery [14]. Various studies have proven better efficiency with AI in implant placement than freehand done by experienced surgeons. With the help of 3D guided stents, implant surgeries have shorter operation time, safer approximation around delicate structures and higher intra operative accuracy. In tumour resection, AI allows more thorough surgical resection potentially decreasing need for revision procedures [15].

\section{Artificial intelligence in dento -maxillofacial radiology}

Radiographic images can be uploaded in the system.AI will detect the pathogenesis by analysing the data. Thus, helping the PR actioner with diagnosis which is related clinically. Integration of CBCT and MRI with AI will ease the radiologist work [16]. 


\section{Other applications in dentistry}

Artificial intelligence based on virtual dental assistants can perform several tasks in the dental clinic with greater precision, fewer errors and less manpower compared to humans. It can be used to coordinate appointments, managing insurance and paper works as well assisting clinical diagnosis or treatment planning. It is very useful in alerting the dentist about patient's medical history as well as habits like alcoholism and smoking. In dental emergencies, the patient has a option of emergency tele assistance especially when the practitioner is unavailable. Thus a detailed virtual database of the patient can be created which will go a long way in providing ideal treatment for the patient.

\section{Smart scheduling appointments}

AI can be used to schedule patient appointments. This will definitely will lessen the burden on assistant and it will be easy to train the new person who is appointed.

\section{Smart patient communication}

AI can also help in Doctor patient communication. Any queries of patient can be answered by AI and if complicated can be directed to the PR actioner. Therefore assistant has to spend less time on handling prescriptions post-op and it will easy to recognize emergencies prior to the appointment AI can provide information to the dental health care professional about any relevant medical history or allergies that the patient may have [2]. For patients it can set up regular reminders who are on tobacco or smoking cessation programs. AI can help the patients when doctor is not available $[17,18]$.

\section{Conclusion}

$\mathrm{AI}$ is growing tremendously but it can never replace human knowledge, skill, and decision ability. As biological systems are much more complex, it's not only diagnosing but relating it to clinical findings and personalised care is also important in comprehensive care. Although AI can assist in numerous ways, final call has to be made by a dentist as dentistry is a multidisciplinary approach.

\section{Bibliography}

1. Brickley MR., et al. "Neural networks: A new technique for development of decision support system in dentistry". Journal of Dentistry 26 (1998): 305-309.

2. Khanna SS and Dhaimade PA. "Artificial Intelligence: Transforming dentistry today". Indian Journal Basic Applied Medical Research 6 (2017):161-167.
3. Luslid LB. "Medical progress - medical electronics". The New England Journal of Medicine 252 (1995): 58-55.

4. Steimann F. "On the use and usefulness of fuzzy sets in medical AI”. Artificial Intelligence in Medicine 21 (2001):131-137.

5. Kim Ey., et al. "Predictive modelling of dental pain using neural network". Studies in Health Technology and Informatics 146 (2009): 745-746.

6. Yaee HT., et al. "Octree-based virtual dental training system with a haptic device". Computer-Aided Design and application 3 (2006): 415-424.

7. Murray T. "Authoring intelligent tutoring system for visual classification problem solving". Artificial Intelligence in Medicine 36 (2006): 85-117.

8. Jae-Joon Hwang., et al. "An overview of deep learning in the field of dentistry". Imaging Science in Dentistry 49.1 (2019): $1-7$.

9. Xie X Wangt and Wang A. "Artificial neural Network Modelling for Deciding if extraction are necessary prior to orthodontic treatment". The Angle Orthodontist 80 (): 262-266.

10. Kattadiyil MT., et al. "Intraoral Scanning of hard and soft tissue for partial removable dental prosthesis fabrication". Journal of Prosthetic Dentistry 112 (2014): 444-448.

11. Hammond P., et al. "logicbased constraints and design of dental prosthesis". Artificial Intelligence in Medicine 5.5 (1993): 431-446.

12. Zivko-Babic., et al. "Estimation of chemical resistance of dental ceramics by neural network". Dental Materials Journal 24 (2008):18-27.

13. Ruppin J., et al. "Evaluation of the accuracy of three different computer aided surgery system in dental implantology: Optical tracking Vessels stereolithograpic splint systems". Clinical Oral Implant Research 19.17 (2008): 709-716.

14. Widman G. "Image guide surgery and medical robotics in cranial area”. Biomedical Imaging and Intervention Journal 3.1 (2007): 1-9.

15. Saement DP., et al. "Stereolithographic surgical templates for placement of dental implants in complex cases". International Journal of Periodontics and Restorative Dentistry 23.3 (2003): 287-295. 
16. Medonce EA. "Clinical decision support systems: perspectives in dentistry". Journal of Dental Education 68 (2014): 589-597.

17. Majumdar B., et al. "Technology: Artifical intelligence". British Dental Journal 224 (2018): 916-917.

18. Bijio Alexzender and Sunil J. "Artificial intelligence in dentistry: current concepts and a peep into the future". International Journal of Advanced Research 6.12 (2018): 1105-1108.

Volume 3 Issue 12 December 2019

(C) All rights are reserved by Amrita Pandita Bhatia and Shilpi Tiwari. 